\title{
Synovial lipomatosis of the metatarsophalangeal joint: A case report
}

\author{
JIANGYINZI SHANG*, FAN ZOU*, MIN DAI, BIN ZHANG and TAO NIE \\ Department of Orthopedics, The First Affiliated Hospital of Nanchang University, \\ Artificial Joints Engineering and Technology Research Center of Jianxi Province, Nanchang, Jiangxi 330006, P.R. China
}

Received November 10, 2014; Accepted July 7, 2015

DOI: $10.3892 / \mathrm{ol} .2016 .4218$

\begin{abstract}
Synovial lipomatosis, also termed lipoma arborescens, is an extremely rare disorder of the synovium that causes joint pain, swelling and effusion. To date, only a small number of cases have been reported in the literature. The current study presents the case of a 44-year-old male with repeated swelling of the metatarsophalangeal joints of the left hallux, which had persisted for $\sim 3$ years. The main clinical manifestations on presentation included multiple osseous lumps and limited activity of the left hallux without pain. Magnetic resonance imaging of the left hallux revealed a mass surrounding the left metatarsophalangeal joints. Subsequently, the lesion was resected. Pathological examination revealed well-defined lobules of mature adipocytes separated by thin fibrous septa, which indicated a diagnosis of synovial lipomatosis of the metatarsophalangeal joint of the left foot. The aim of this study was to evaluate synovial lipomatosis of the metatarsophalangeal joint, with an analysis of the clinical parameters and pathological features of the disorder.
\end{abstract}

\section{Introduction}

Adipose tissue is widely distributed throughout the human body. Thus, lipomas may originate almost anywhere in the body and represent one of the most common benign neoplasms of the soft tissues (1). Synovial lipomatosis, which derives its name from Hoffa's disease, is also known as villous lipomatous proliferation of the synovium or lipoma arborescens (2). Synovial lipomatosis is a rare disorder of the synovium, which results in joint pain, swelling and effusion, and to date only a small number of cases have been reported in the literature (3-5). The disease is generally identified in the knee

Correspondence to: Dr Tao Nie, Department of Orthopedics, The First Affiliated Hospital of Nanchang University, Artificial Joints Engineering and Technology Research Center of Jianxi Province, 17 Yong Wai Zheng Jie, Nanchang, Jiangxi 330006, P.R. China E-mail: ncnietao@163.com

*Contributed equally

Key words: synovial lipomatosis, metatarsophalangeal joint, lipoma arborescens joints, with a lower predilection for other joints (4), such as the elbows, shoulders and wrists (5). However, cases of synovial lipomatosis in the hindfoot (6) and peroneal tendon sheaths (7) have been reported. No cases of synovial lipomatosis in the metatarsophalangeal joints have been reported thus far. In the present study, a case of synovial lipomatosis occurring in the metatarsophalangeal joints of the left hallux is presented, and the results of imaging and histological examinations are discussed. Written informed consent was obtained from the patient for the publication of this study.

\section{Case report}

A 44-year-old male presented to The First Affiliated Hospital of Nanchang University (Nanchang, China) in August 2011 with recurrent swelling of the metatarsophalangeal joints of the left hallux, which had persisted for $\sim 3$ years. The patient had found a mass surrounding the left hallux 3 years prior to presentation, which had gradually increased in size. The patient had no history of joint disorders, trauma or general disease. Upon physical examination, a non-tender, boggy soft-tissue mass was palpable on the metatarsophalangeal joints of the left hallux (Fig. 1). The mass was soft, mobile and well-defined. No erythema was identified, the area was not hot to touch and the blood supply to the left foot was normal. Laboratory routine blood tests were also negative. B-mode ultrasonography performed at another hospital prior to admission to The First Affiliated Hospital of Nanchang University showed a thickened soft-tissue lesion surrounding the metatarsophalangeal joints, which was diagnosed as chronic synovial hyperplasia.

An amorphous tumor surrounding the first metatarsal bone of the left hallux was identified on magnetic resonance imaging (MRI) (Fig. 2), which exhibited intermediate signal intensity on T1-weighted images (Fig. 2A) and a signal intensity similar to that of subcutaneous adipose tissue on T2-weighted images (Fig. 2B). These findings were consistent with a diagnosis of a giant cell tumor of the tendon sheath. In addition, no evident abnormalities of left foot bone signals were identified. The lesion was completely resected and sent for histopathological examination.

Histopathological analysis of the resected tissue revealed a hoary, soft, nodular tissue mass, which was $7 \times 5 \times 2 \mathrm{~cm}$ in size (Fig. 3A). Pathological examination revealed well-defined lobules of mature adipocytes separated by fibrous septa and 

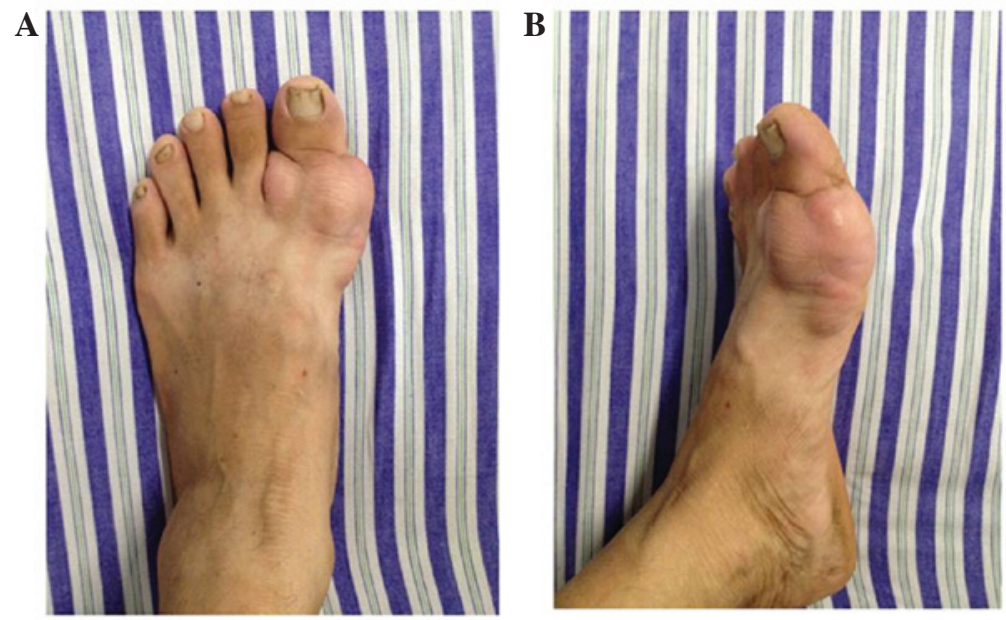

Figure 1. Generalized soft-tissue swelling surrounding the left metatarsophalangeal joint. (A) Dorsal and (B) medial views.
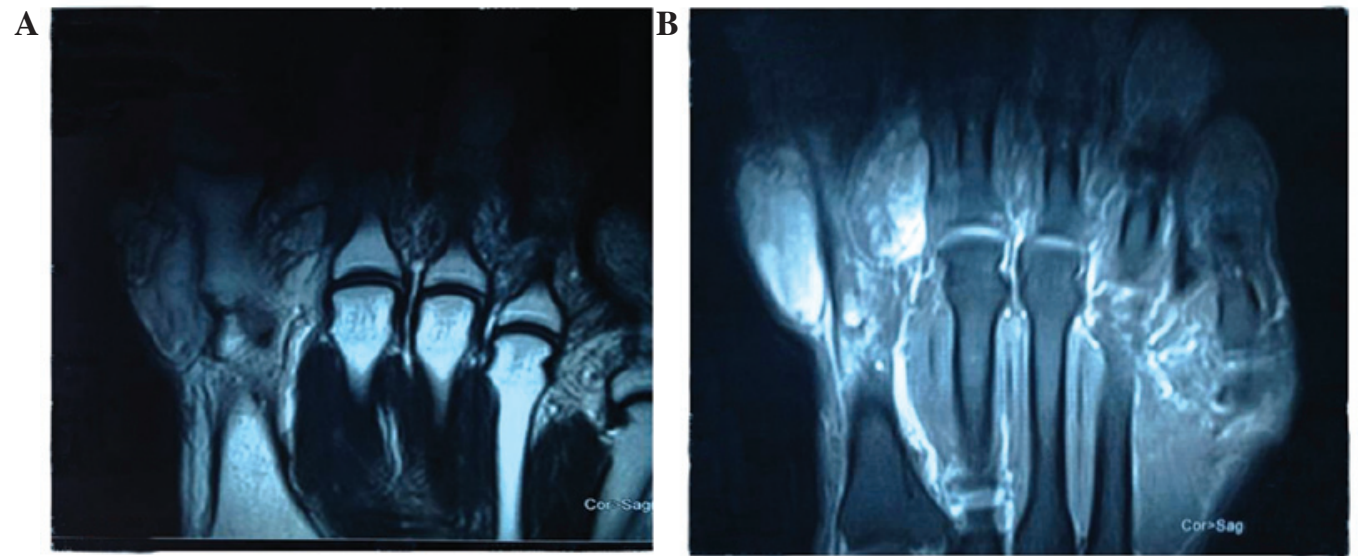

Figure 2. (A) T1-weighted MRI sequence showing a bilateral mass-like lesion. (B) T2-weighted MRI sequence revealing a lesion surrounding the metatarsophalangeal joints with fat high signal intensity. MRI, magnetic resonance imaging.
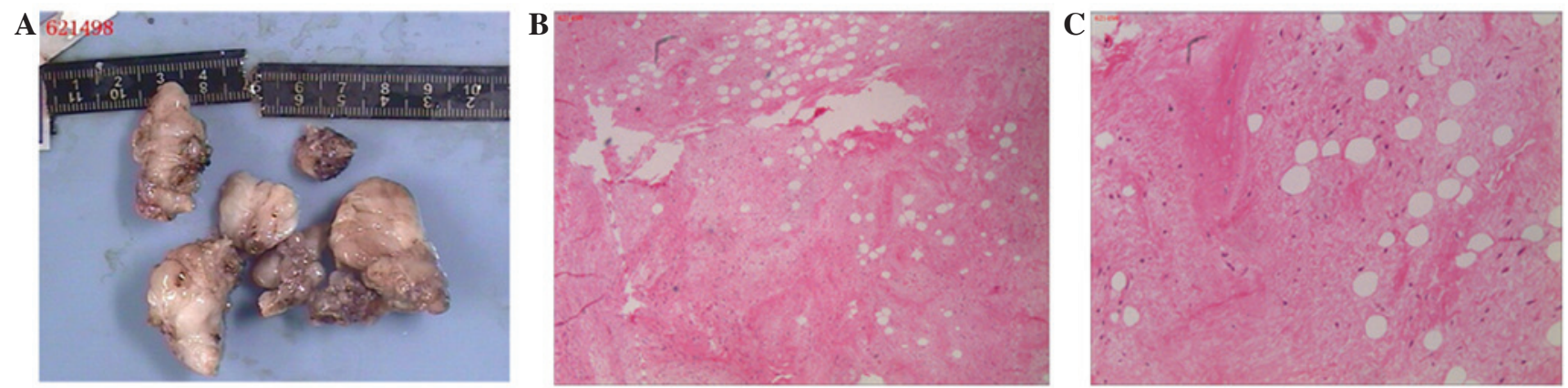

Figure 3. (A) Excised mass (7x5x2 cm in size) and the fibrous capsule enclosing mature adipose tissue. (B) Normal synovial cells covering mature adipose tissue with mild fibrosis (hematoxylin and eosin staining; magnification, $\mathrm{x} 40$ ). (C) Chronic inflammation is evident with scattered inflammatory cells (hematoxylin and eosin staining; magnification, x100).

covered by synovial lining (Fig. 3B), and extensive proliferation of the fibrous and adipose tissues, with infiltration of chronic inflammatory cells (Fig. 3C). The color of the neoplasm was different to the yellow tissue normally observed with giant cell tumors of the tendon sheath. Thus, based on the results of pathological analysis, a final diagnosis of synovial lipomatosis was established. A follow-up examination two years after surgery revealed no disease recurrence and the patient exhibited good hallux function.

\section{Discussion}

Lipoma, which exhibits no gender predilection, is a common tumor-like lesion of the synovium that accounts for $\sim 50 \%$ 
of soft-tissue tumors (8). According to the previously published literature, synovial lipomatosis most commonly occurs in older individuals, with a median age of 50 years (range, 39-66 years) (9). Although the etiology of synovial lipomatosis remains unclear, a high body mass index appears to exhibit a vital role in cases of short bowel syndrome with synovial lipomatosis in multiple joints (10), and the clinical manifestations are usually a result of the mass, and include pain, crepitus, limitation of motion and joint effusion (1). The diagnosis of synovial lipomatosis is achieved primarily by MRI, using adipose tissue-suppressed sequences in particular; synovial lipomas and adipose tissue exhibit similar high signal intensities on T1- and T2-weighted images $(1,11)$. As their treatment and prognosis differ, it is important to differentiate synovial lipoma from other adipose tissue proliferative diseases, including pigmented villonodular synovitis, synovial chondromatosis, synovial hemangiomatosis and rheumatoid arthritis (12). Pigmented villonodular synovitis exhibits diffuse signals of low intensity on T1- and T2-weighted images. Synovial chondromatosis varies from low to high signal on T2- and T1-weighted sequences according to the cartilaginous components of the lesion. Synovial hemangiomas exhibit intermediate signal intensity on T1- and T2-weighted images, with areas of high signal intensity due to the presence of fibrous septa between the vascular channels and adipose tissue in the lesion. Rheumatoid arthritis exhibits intermediate to low signal intensity on T1- and T2-weighted images, and is associated with the formation of fibrous pannus (12).

Surgical treatments for synovial lipomatosis include arthroscopy and excision (5), and treatment choice is dependent on the extent of involvement. Arthroscopy is the preferred treatment choice for cases of synovial lipomatosis, particularly in larger joints that exhibit low recurrence rates, as it is minimally invasive with good recovery rates $(5,13)$. However, in certain cases, more extensive surgeries, including arthrotomy and synovectomy, may be required $(14,15)$. The histomorphology of synovial lipomatosis is associated with adipocyte metaplasia and inflammation, and fibrosis (16); thus, synovial lipomatosis can be more accurately described as the process of overgrowth and infiltration of mature adipose tissue within the synovium. Occasionally, fibrous septa may be visible between the vascular channels and adipose tissue within the lesion (17).

In the present case, the pre-operative clinical manifestation and clinical examination indicated a diagnosis of a giant cell tumor of tendon sheath. However, the resected surgical specimens were gray, whereas the sheaths of giant cell tumors are usually pale yellow, and thus, based on the results of MRI and the pathological examination, the tumor was diagnosed as a synovial lipoma.

In conclusion, synovial lipomatosis is an extremely rare lesion of the synovium that is considered to occur as a result of inappropriate adipose tissue deposition and degenerative articular diseases of the joints. In this study, a rare case of synovial lipomatosis involving the metatarsophalangeal joints was presented. Increased understanding with regard to the characteristic MRI features and distinct histomorphology of synovial lipomatosis may lead to advances in the diagnosis of this rare disease.

\section{Acknowledgements}

This study was supported by the Gan-Po Talents Project 555 of Jiangxi Province.

\section{References}

1. Zhu W, Wang W, Chen Y and Xiao T: Synovial lipoma in intra-patellar fat pad of the knee joint. Pak J Med Sci 28: 228-230, 2012.

2. Saddik D, McNally EG and Richardson M: MRI of Hoffa's fat pad. Skeletal Radiol 33: 433-444, 2004.

3. Pudlowski RM, Gilula LA and Kyriakos M: Intraarticular lipoma with osseous metaplasia: Radiographic-pathologic correlation. AJR Am J Roentgenol 132: 471-473. 1979.

4. Bejia I, Younes M, Moussa A, Said M, Touzi M and Bergaoui N: Lipoma arborescens affecting multiple joints. Skeletal Radiol 34: 536-538, 2005.

5. Yildiz C, Deveci MS, Ozcan A, Saraçoğlu HI, Erler K and Basbozkurt M: Lipoma arborescens (diffuse articular lipomatosis). J South Orthop Assoc 12: 163-166, 2003.

6. Jowett C, Mitra P, O'Donnell P and Singh DS: Synovial lipomatosis of hindfoot tendon sheaths: Case reports and literature review. Foot Ankle Int 29: 752-755, 2008.

7. Vinson EN, Dodd LG, Merian M and Martinez S: Synovial lipomatosis arborescens of the peroneal tendon sheath. Skeletal Radiol 37: 947-950, 2008.

8. S Amarjit K, Budhiraja S, Chandramouleeswari K and Anita S: Knee locking in osteoarthritis due to synovial lipoma: A case report. J Clin Diagn Res 7: 1708-1709, 2013.

9. Kakkar N, Vasishta RK and Anand H: Pathological case of the month. Synovial lipomatosis. Arch Pediatr Adolesc Med 153: 203-204, 1999.

10. Siva C, Brasington R, Totty W, Sotelo A and Atkinson J: Synovial lipomatosis (lipoma arborescens) affecting multiple joints in a patient with congenital short bowel syndrome. J Rheumatol 29: 1088-1092, 2002.

11. Ku JH, Cho HL, Park JT, Wang TH and Yang HS: Intra-articular synovial lipoma in the posteromedial compartment of the knee. Arthrosc Orthop Sports Med 1: 54-58, 2014

12. Vilanova JC, Barceló J, Villalón M, Aldomà J, Delgado E and Zapater I: MR imaging of lipoma arborescens and the associated lesions. Skeletal Radiol 32: 504-509, 2003.

13. Hirano K, Deguchi M and Kanamono T: Intra-articular synovial lipoma of the knee joint (located in the lateral recess): A case report and review of the literature. Knee 14: 63-67, 2007.

14. Ren F and Li Z: Diagnosis and therapy of synovial lipomatosis of the knee joint. Chin J Orthop 29: 539-543, 2009.

15. Haasbeek JF and Alvillar RE: Childhood lipoma arborescens presenting as bilateral suprapatellar masses. J Rheumatol 26: 683-686, 1999.

16. Franco M, Puch JM, Carayon MJ, Bortolotti D, Albano L and Lallemand A: Lipoma arborescens of the knee: Report of a case managed by arthroscopic synovectomy. Joint Bone Spine 71: 73-75, 2004.

17. Rao S, Rajkumar A, Elizabeth MJ, Ganesan V and Kuruvilla S: Pathology of synovial lipomatosis and its clinical significance. J Lab Physicians 3: 84-88, 2011. 